\title{
A unified tabu search algorithm for vehicle routing problems with soft time windows
}

\author{
$\mathrm{Z} \mathrm{Fu}{ }^{1 *}, \mathrm{R}_{\text {Eglese }}^{2}$ and $\mathrm{LYO} \mathrm{Li}^{3}$ \\ ${ }^{1}$ Central South University, Changsha, P.R. China; ${ }^{2}$ Lancaster University Management School, Lancaster, \\ UK; and ${ }^{3}$ The Hong Kong Polytechnic University, Hong Kong, P.R. China
}

The different ways of allowing time window violations lead to different types of the vehicle routing problems with soft time windows (VRPSTW). In this paper, different types of VRPSTW are analysed. A unified penalty function and a unified tabu search algorithm for the main types of VRPSTW are presented, with which different types of VRPSTW can be solved by simply changing the values of corresponding parameters in the penalty function. Computational results on benchmark problems are provided and compared with other methods in the literature. Some best known solutions for the benchmark problems in the literature have been improved with the proposed algorithm.

Journal of the Operational Research Society (2008) 59, 663-673. doi:10.1057/palgrave.jors.2602371

Published online 7 February 2007

Keywords: distribution; vehicle routing; soft time windows; heuristics; tabu search

\section{Introduction}

The basic vehicle routing problem (VRP) calls for the determination of a set of minimum-cost routes to be performed by a fleet of vehicles to serve a given set of customers with known demands, where each route originates and terminates at a single depot. The total cost consists of two parts, which are the objectives to be minimized: (1) the total number of routes (vehicles) required to serve all customers and (2) the total vehicle travel distance of all routes. It is assumed that the capital cost of an additional vehicle will always exceed any travelling cost that could be saved by its use. Therefore, the priority is given to the first objective. Each customer must be assigned to only one vehicle and the total demand of all customers assigned to a vehicle does not exceed its capacity.

The vehicle routing problem with time windows (VRPTW) is an extension of the basic VRP in which vehicle capacity constraints are imposed and each customer $i$ is associated with a time interval $\left[a_{i}, b_{i}\right]$, called a time window, during which service must begin. In any vehicle route, the vehicle may not arrive at customer $i$ after $b_{i}$ to begin service. If a vehicle arrives before $a_{i}$, it waits. When the time window requirements are strictly enforced, the problem is also called a VRP with hard time windows (VRPHTW). The problem may arise in a variety of applications, including retail distribution, school bus routing, bank deliveries, mail and newspaper deliveries, municipal waste collection, fuel oil deliveries, and more.

\footnotetext{
*Correspondence: Z Fu, School of Traffic and Transportation Engineering, Central South University at Railway Campus, Changsha, Hunan 410075, P.R. China.

E-mail: zhfu@mail.csu.edu.cn
}

The time windows are designed to handle issues such as availability of personnel to load or unload the vehicle, traffic regulations, and customer preferences. For example, a store may only accept deliveries before it opens for business at 9:00 a.m. For VRPHTW, research has flourished over the last two decades. The interested reader will find excellent surveys in Cordeau et al (2002).

The vehicle routing problem with soft time windows (VRPSTW) is an extension of VRPHTW in which some or all customer time window requirements are not strictly enforced and can be violated by paying appropriate penalties. For each customer $i$, we can define penalty functions to calculate the penalty payable if the vehicle arrives before $a_{i}$ or after $b_{i}$. If a certain customer's time window cannot be violated, that is, it is hard, the penalty payable to that customer for any violation is set to infinity. In that respect, the VRPHTW is a special case of the VRPSTW in which no violations are allowed. There are many good reasons for allowing the time windows to be soft, as stated in Koskosidis et al (1992), Balakrishnan (1993), Taillard et al (1997), Fagerholt (2001), and Chiang and Russell (2004), including:

(1) Many applications do not require a time window that is precise to exact points of time. Therefore, time windows are usually soft by nature.

(2) It is usually impossible to determine accurate vehicle travel times in practice.

(3) Setting the time windows to be soft may allow significant savings in the number of vehicles required and/or the total vehicle travel distance of all routes to be achieved.

(4) The VRPSTW model is more general and includes the VRPHTW. An algorithm for the VRPSTW can be 
extended to solve the VRPHTW by appropriately raising the penalty coefficients.

(5) VRPSTW can always generate feasible solutions in instances where a hard time window approach would have failed. Problems with hard time windows and a small fleet might not have a solution satisfying all customers. In this case, the VRPSTW model would yield usable solutions where some of the customers would not be serviced within the desired time windows.

(6) The VRPSTW model can be used to find a good trade-off between fleet size and service quality to customers.

Note that the objectives to be minimized for the VRPSTW become three: (1) the total number of routes (vehicles) required to serve all customers, (2) the total penalty of time window violations, and (3) the total vehicle travel distance of all routes.

The VRPSTW has received some attention over the last decade. Koskosidis et al (1992) presented an optimizationbased heuristic to solve the problem. Balakrishnan (1993) described three simple heuristics for the VRPSTW, which were based on nearest neighbour, Clarke-Wright savings, and space-time rules, respectively. Besides these classical heuristics, Taillard et al (1997) designed a tabu search (TS) heuristic for a special case of the VRPSTW, in which the total number of vehicles required was given and fixed. This meant that the fleet size was not a decision variable. Therefore, the objective (1) mentioned above did not need to be minimized. Fagerholt (2001) described a real ship scheduling problem with soft time windows and proposed an optimization-based approach based on a set partitioning formulation to solve the problem. Recently, Chiang and Russell (2004) developed a TS solution method for the type of VRPSTW that Balakrishnan (1993) studied.

In this paper, we present a unified penalty function and a unified TS algorithm for the different types of VRPSTW. Experimental results are reported and compared with other approaches.

\section{Notation and problem representation}

Let $G=(V, E)$ be a given undirected network, where $V=\{0, \ldots, n\}$ is the vertex set and $E$ is the edge set. Vertices $i=1, \ldots, n$ correspond to the customers, whereas vertex 0 corresponds to the depot.

A non-negative cost, $c_{i j}$, is associated with each edge $(i, j) \in E$ and represents the travel time (distance) spent from vertex $i$ to vertex $j$. If vehicle $k$ travels directly from vertex $i$ to vertex $j$, then $x_{i j k}=1$, otherwise $x_{i j k}=0$. A complete network is assumed. If any edges between vertices are missing in the original graph, then they are replaced by edges with an artificially high cost.

Each customer $i(i=1, \ldots, n)$ is associated with a known non-negative demand, $d_{i}$, to be delivered or picked up. A set of identical vehicles, each with capacity $C$, is available at the depot. To ensure feasibility, we assume that $d_{i} \leqslant C$ for each $i=1, \ldots, n$. Each vehicle may serve at most one route. Each vehicle route must start and end at the depot.

Furthermore, each customer $i(i=1, \ldots, n)$ is associated with a time window $\left[a_{i}, b_{i}\right]$ during which service should ideally begin and a service time $s_{i}$ for unloading or loading the goods. The depot has a service time $s_{0}=0$, and a time window $\left[a_{0}, b_{0}\right]$. Normally, $a_{0}=0$. The start time of each vehicle route is greater than or equal to $a_{0}$. Moreover, observe that the time window requirements induce an implicit orientation of each route even if the original matrices are symmetric, and an implicit route length constraint (ie a latest time instant to complete the route) where the maximum route length $L=b_{0}$. Each route length consists of the vehicle travel time, waiting time at some customers, and time to serve all customers on the route. Therefore, the length of route $k$ is

$$
\sum_{i=0}^{n} \sum_{j=0}^{n} c_{i j} x_{i j k}+\sum_{i \in N_{k}} w_{i}+\sum_{i \in N_{k}} s_{i}
$$

where $w_{i}$ is the waiting time at customer $i$ and $N_{k}$ is the set of customers assigned to route $k$.

In the hard time window case, a vehicle is not allowed to arrive at customer $i$ after $b_{i}$ to begin service. If a vehicle arrives before $a_{i}$, it waits. However, in the soft time window case, each customer time window can be violated by paying an appropriate penalty. Let $t_{i}$ denote the arrival time instant at customer $i$; the penalty payable $P\left(t_{i}\right)$ can be represented as a linear function of the amount of time window violation. For customer $i$, penalty coefficients $\alpha_{i}$ and $\beta_{i}$ are defined to denote the penalty payable for each time unit of service beginning before $a_{i}$ and after $b_{i}$, respectively. In practice, these coefficients could represent the costs of lost sales, goodwill, etc, due to the customer inconvenience for not meeting the time windows, and are therefore often called inconvenience costs. It is not necessary for $\alpha_{i}$ and $\beta_{i}$ to be equal, or for these penalty coefficients to be equal across customers. For some important customers or customers whose time window requirements are very strict, the value of $\alpha_{i}$ and $\beta_{i}$ can be greater, even set to infinity which, in effect, converts this time window to a hard time window.

The different ways of allowing time window violations lead to different types of VRPSTW.

Type 1: If a vehicle arrives before $a_{i}$, it waits, as in the hard time window case. But a vehicle is allowed to arrive at customer $i$ after $b_{i}$ to begin service by paying an appropriate penalty, see Figure 1(a). Taillard et al (1997) proposed a TS heuristic for this type of problem.

Type 2: Both early and late service at customer locations are allowed by paying appropriate penalties, see Figure 1(b). Koskosidis et al (1992) presented an optimization-based heuristic for this type of problem. 


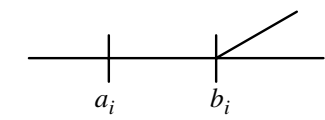

d

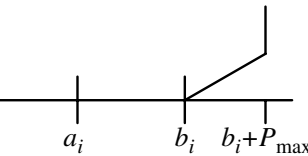

b
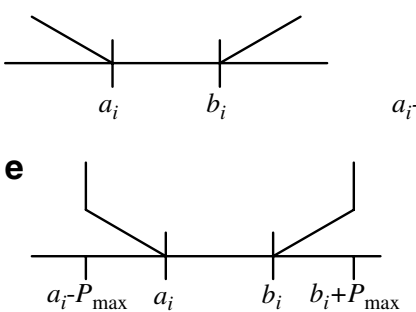

C

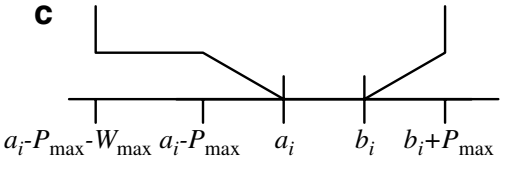

f

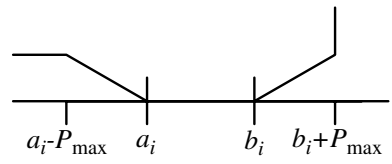

Figure 1 Main types of VRPSTW. In each diagram, the horizontal axis represents time and the vertical axis represents the penalty cost.

Type 3: Assume the maximum allowable violation of the time windows to be $P_{\max }$. Then the outer time window of each customer $i$ may be enlarged to $\left[a_{i}-P_{\max }, b_{i}+\right.$ $\left.P_{\max }\right]$. Furthermore, assume that the maximum waiting time allowed before the outer time window is $W_{\max }$ (see Figure 1(c)). This is the type of the problem that Balakrishnan (1993) and Chiang and Russell (2004) studied. Balakrishnan (1993) described three simple heuristics for it. Chiang and Russell (2004) developed a TS solution method for the problem.

Type 4: Based on Type 1, define $P_{\max }$ as the maximum allowable lateness at customer locations, see Figure 1(d).

Type 5: Based on Type 2, define $P_{\max }$ as the maximum allowable violation of the time windows. Then the outer time window of each customer $i$ may be enlarged to $\left[a_{i}-P_{\max }, b_{i}+P_{\max }\right]$, see Figure 1(e). The time for the start of service at customer $i$ must be within the outer time window $\left[a_{i}-P_{\max }, b_{i}+P_{\max }\right]$ and preferably within the inner time window $\left[a_{i}, b_{i}\right]$. The case study by Fagerholt (2001) belongs to this type of problem, in which an optimization-based approach based on a set partitioning formulation was proposed to solve the problem.

Type 6: Based on Type 3, remove the limitation of the maximum allowable waiting time $W_{\max }$, see Figure 1(f).

Some other variants can be defined on the base of the above main types of VRPSTW. If we let $E_{\max }$ and $L_{\max }$ represent the maximum allowable violation of time windows before $a_{i}$ and after $b_{i}$, respectively, instead of $P_{\max }$, then a unified penalty function for the above main types of VRPSTW can be defined as

$$
P\left(t_{i}\right)= \begin{cases}\infty(\text { infeasible }) & \text { if } t_{i}<a_{i}-E_{\max }-W_{\max } \\ \alpha_{i} E_{\max } & \text { if } a_{i}-E_{\max }-W_{\max } \leqslant t_{i} \\ & <a_{i}-E_{\max } \\ \alpha_{i}\left(a_{i}-t_{i}\right) & \text { if } a_{i}-E_{\max } \leqslant t_{i}<a_{i} \\ 0 & \text { if } a_{i} \leqslant t_{i} \leqslant b_{i} \\ \beta_{i}\left(t_{i}-b_{i}\right) & \text { if } b_{i}<t_{i} \leqslant b_{i}+L_{\max } \\ \infty(\text { infeasible }) & \text { if } t_{i}>b_{i}+L_{\max }\end{cases}
$$

where $\alpha_{i}$ and $\beta_{i}$ are the penalty coefficients. $P\left(t_{i}\right)$ can represent the penalty function for different types of VRPSTW as different values are assigned to $W_{\max }, E_{\max }$, and $L_{\max }$. When $W_{\max }=\infty, E_{\max }=0$, and $L_{\max }=\infty$, it is for Type 1 . When $W_{\max }=0, E_{\max }=\infty$, and $L_{\max }=\infty$, it is for Type 2 . When $0<W_{\max }$ and $E_{\max }, L_{\max }<\infty$, it is for Type 3 . When $W_{\max }=\infty, E_{\max }=0$, and $0<L_{\max }<\infty$, it is for Type 4 . When $W_{\max }=0,0<E_{\max }$, and $L_{\max }<\infty$, it is for Type 5 . When $W_{\max }=\infty, 0<E_{\max }$, and $L_{\max }<\infty$, it is for Type 6 . Furthermore, when $W_{\max }=\infty$ and $E_{\max }=L_{\max }=0$, it is for the VRPHTW.

As mentioned in the Introduction, the VRPSTW has three objectives to be minimized. Wishing to find a good trade-off between cost and service quality to customers, like Balakrishnan (1993), we judge the quality of the solution obtained using the following criteria in decreasing order of importance: (1) the total number of vehicles used, (2) the total deviation of time window to start service, and (3) the total vehicle travel distance. Therefore, a feasible solution with a certain number of vehicles always dominates over any other feasible solutions requiring more vehicles. This in fact introduces a hierarchical objective function: first, minimize objective (1) and then, for the same number of vehicles, minimize objectives (2) and (3). The objective function can be expressed in pre-emptive goal-programming notation as

$$
\operatorname{Min} z=P_{1} K+P_{2}\left[\sum_{i=1}^{n} P\left(t_{i}\right)+\sum_{i=0}^{n} \sum_{j=0}^{n} \sum_{k=1}^{K} c_{i j} x_{i j k}\right]
$$

where $K$ represents the total number of vehicles used, and $P_{1}$ and $P_{2}$ denote priority levels $\left(P_{1}>>P_{2}\right)$. Since objective (2), as stated above, is more important than objective (3), then the level of the importance can be adjusted by the penalty (weight) coefficients $\alpha_{i}$ and $\beta_{i}$.

\section{The tabu search algorithm}

Our algorithm is based on tabu search, a local search metaheuristic introduced first by Fred Glover in 1986, and since then has been used to solve many practical applications. It is designed to guide the solution process to escape local optima. A thorough discussion may be found in Glover and Laguna (1997). 
We developed an effective TS heuristic for the open vehicle routing problem (OVRP) in a previous work (Fu et al, 2005, 2006). The full details of the algorithm were given in the referenced paper and so only the outline of the algorithm and the changes that have been made to apply it to the VRPSTW are described here.

\section{Initial solution}

An initial solution is required for any TS algorithm to start the local search process. With respect to the TS metaheuristic for the VRP, some researchers, such as Van Breedam (2001) and Brandão (2004), claimed that the performance of the TS heuristic was highly dependent on the quality of the initial solutions. However, in our previous work (Fu et al, 2005), it was shown that the initial solution did not have a large influence on the final solutions in the proposed TS heuristic. In this paper, therefore, the initial solution is generated by building up successive routes where the next customer is chosen at random and added to the end of the route unless this violates the capacity or route length constraints; in that case the route is completed back to the depot and a new route starts.

\section{Neighbourhood structure}

Some discussion of different neighbourhood structures for VRP was presented in Fu et al (2005), including proposals from Pureza and França (1991), Osman (1993), Gendreau et al (1994), Duhamel et al (1997), Cordeau et al (2001), Brandão (2004) and Taillard et al (1997).

The TS algorithm presented in this paper uses the same mixed neighbourhood structure as was introduced in $\mathrm{Fu}$ et al (2005), but some necessary modification is made for the VRPSTW where each vehicle route must start and end at the depot. There are four different types of move that may be used in this mixed neighbourhood structure, and the algorithm selects a type of move randomly at each iteration. The four different types of move are based on the 2-interchange mechanism, but differ in the details of the moves that are implemented. The four different types of move are referred to as vertex reassignment, vertex swap, 2-opt and 'tails' swap. Some of the possible transformations are described in the following paragraphs.

A solution of the problem can be denoted by a permutation $\left(0, i_{1}, i_{2}, i_{3}, 0, i_{4}, \ldots, 0, i_{n-1}, i_{n}, 0\right)$ of $(0, \ldots, n)$. Only 0 is allowed to appear more than once, each time for a new route. The first 0 and the following vertices before the second 0 consist of the first delivery route, the second 0 and the following vertices before the third 0 consist of the second delivery route, and so on. In consideration of feasibility, the first as well as the last item of a solution must be 0 . If one 0 is adjacent to another, that means one route contains no customer, so it can be eliminated.

Select two different vertices (customer or depot, within the same route or different ones) randomly. Examples are shown underlined below. Perform one of the following four moves randomly.

(a) Vertex reassignment: Remove the first selected vertex from its current position on the route and insert it into the position after the second selected vertex, that is,

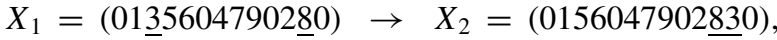
$X_{1}=(0135 \underline{064709280}) \rightarrow X_{2}=(0135647092800)$.

(b) Vertex swap: Exchange the positions of two selected vertices, that is, $X_{1}=(0135 \underline{06} \underline{4790280)} \rightarrow$ $X_{2}=(0135 \underline{4} 6 \underline{0790280}), X_{1}=(0135 \underline{6} 0479 \underline{0280}) \rightarrow$ $X_{2}=(0135 \underline{0} 0479 \underline{6} 280)$.

(c) 2-opt: Reverse the order of all elements between two selected vertices like the standard 2-opt move in TSP, if two selected vertices are within the same route, that is, $X_{1}=\left(01 \underline{3} 56 \underline{40790280)} \rightarrow X_{2}=(01 \underline{465} \underline{3} 0790280)\right.$, $X_{1}=(0135604790280) \rightarrow X_{2}=(0135697400280)$.

(d) 'Tails' swap: Exchange the 'tails' after two selected vertices (from the selected vertex to the end of the route; both vertices must be customers), if two selected vertices are in different routes, that is, $X_{1}=(0135604790280) \rightarrow$ $X_{2}=(013 \underline{7904} \underline{560280})$.

The first and the last 0 of a solution are not allowed to be selected and removed during this 2-interchange process. Note that some moves only make small changes to the current solution and carry on the search within a restricted part of the solution space, facilitating the algorithm to converge; some moves make larger changes to the current solution and guide the search to different areas of the solution space.

(a)-(c) are the moves that can possibly reduce the number of routes. The 'tails' swap or move (d) was originally introduced because of the structure of the OVRP, where we wished to preserve the 'tails' or final parts of routes which would typically include customers far from the depot, while allowing the set of customers included in the early part of the route to be changed. However, this type of move has been retained for the VRPSTW since it allows changes while preserving the sequence of customers in the earlier and later parts of routes, a feature that may be beneficial when time window requirements are an important feature.

To test the influence of the neighbourhood structure and the search strategy on the performance of the algorithm, we compared the following three cases:

(a) Single type of neighbourhood structure (that is, with only one type of move).

(b) Mixed neighbourhood structure with the above four types of move, and perform all of the four moves one by one for the selected two different vertices.

(c) Mixed neighbourhood structure with the above four types of move, but perform one of the four moves randomly for the selected two different vertices.

It showed that case (3) usually produced better solutions than the other cases did. Therefore, the mixed neighbourhood 
structure and the search strategy in which the current move is randomly selected among the four moves are adopted in this TS algorithm.

\section{Evaluation of solutions}

To facilitate the exploration of the search space, a move is allowed even if it results in an infeasible solution in terms of the vehicle capacity or route length. The extent of infeasibility can be measured by incorporating the vehicle capacity and maximum route length constraints into the objective function by adding a penalty if the constraints are broken. So a solution can be evaluated by the following objective function

$$
\begin{aligned}
\operatorname{Min} z= & P_{1} K+P_{2}\left\{\sum_{i=1}^{n} P\left(t_{i}\right)+\sum_{k=1}^{K}\right. \\
& {\left.\left[\sum_{i=0}^{n} \sum_{j=0}^{n} c_{i j} x_{i j k}+p\left(E_{c}(k)+E_{t}(k)\right)\right]\right\} }
\end{aligned}
$$

where $E_{c}(k)$ and $E_{t}(k)$ are the excess of load and duration in route $k$, respectively, and $p$ is the infeasible penalty coefficient. $E_{c}(k)$ and $E_{t}(k)$ are equal to zero for all routes if a solution is feasible. $p \in[0.00001,200000]$ is initially equal to 1 and weighted by a self-adjusting parameter: every 10 iterations, it is divided by 2 if all 10 previous solutions were feasible or multiplied by 2 if all were infeasible. This mechanism was also used in Fu et al (2005), and is based on the method used by Gendreau et al (1994) in the Tabu route algorithm for the VRP.

\section{Tabu list and stopping criterion}

The tabu list and stopping criterion also follow the method described in $\mathrm{Fu}$ et al (2005). The tabu list contains the move attributes of solutions during the last 5-10 (selected randomly) iterations. The search is terminated if either a specified number of iterations have elapsed in total or since the last best solution was found.

\section{TS algorithm}

The following variables are used in the description of the TS algorithm:

$\begin{array}{ll}\begin{array}{l}\text { iter } \\ \text { max_iter }\end{array} & \begin{array}{l}\text { current number of iterations; } \\ \text { cons_iter }\end{array} \\ & \begin{array}{l}\text { maximum number of iterations; } \\ \text { without any improvement to the best solu- }\end{array} \\ & \text { tion so far; } \\ \text { max_cons_iter } & \text { maximum number of consecutive iterations } \\ & \text { without any improvement to the best solu- } \\ & \text { tion so far; }\end{array}$

cand_list max_cand_list maximum number of candidate moves on the list.

The unified TS algorithm for VRPSTW is described below.

Set $W_{\max }, E_{\max }, L_{\max }, \alpha_{i}$ and $\beta_{i}$ to the corresponding value according to the type of VRPSTW to be solved and the level of importance between objectives (2) and (3).

Generate an initial feasible solution randomly, and set this solution as the current solution and the best solution so far;

Set iter and cons_iter to 0;

While (iter $\leqslant$ max_iter) and (cons_iter $\leqslant$ max_cons_iter)

do

Begin

While (cand_list $\leqslant$ max_cand_list) do
begin

Select two vertices randomly;

Perform one of the four types of neighbourhood move randomly;

Add the solution produced by the selected move to the candidate list;

end;

Select the best solution in the candidate list if it is not tabu, or it produces a solution strictly

better than the best solution so far;

Set the new solution as the current solution, update the tabu list and increment iter;

If the new solution improves the best solution so far, update the best solution so far, and set

cons_iter to 0; Otherwise, increment cons_iter;

End.

This style of TS algorithm was found to be effective for the OVRP in Fu et al (2005), which is why it has been used as the basis for solving the VRPSTW in this paper. Its simple but powerful mixed neighbourhood structure and the stochastic elements in the method allow effective diversification within a local search framework.

\section{Computational results and comparison}

This unified TS algorithm was coded in Delphi 7.0 and implemented on a Pentium-II PC running on $600 \mathrm{MHz}$ with $184 \mathrm{MB}$ RAM. To show the computational performance of the algorithm, it was tested on the 56 benchmark problems generated by Solomon (1987) (can be downloaded from: http://w.cba.neu.edu/ msolomon/problems.htm) and the results were compared with others in the literature.

These test problems were extensively used to test algorithms both for the VRPHTW and for the VRPSTW. In these 100-customer Euclidean problems, the travel times are equivalent to the corresponding Euclidean distances. Six different sets of problems are defined, namely $\mathrm{R} 1, \mathrm{C} 1, \mathrm{RC} 1, \mathrm{R} 2, \mathrm{C} 2$, and $\mathrm{RC} 2$. In problem sets $\mathrm{R} 1$ and $\mathrm{R} 2$, customers are randomly distributed, whereas in sets $\mathrm{C} 1$ and $\mathrm{C} 2$, they are clustered; and a mix of random and clustered structure is contained 
in problem sets $\mathrm{RC} 1$ and $\mathrm{RC} 2$. In problem sets $\mathrm{R} 1, \mathrm{C} 1$ and $\mathrm{RC} 1$, the time window is narrow at the central depot so that only a few customers can be served on each route. The time window is wider for problem sets $\mathrm{R} 2, \mathrm{C} 2$, and $\mathrm{RC} 2$ so that many customers can be visited on each route and only a few vehicles are required.

Our unified TS algorithm for three of the six main types of VRPSTW that were previously defined (Type 1, Type 2, and Type 3) was tested on the benchmark problems in the literature. The best solutions produced by the algorithm during the course of multiple experiments were compared with those produced by other approaches in the literature, using the criteria of judging the quality of the solution in decreasing order of importance: (1) the total number of vehicles used, (2) the total deviation of time window to start service, and (3) the total vehicle travel distance.

The results were produced with the following bounds: max_cand_list $=350$, max_cons_iter $=5000$, and max_iter $=15000$.

\section{Comparison of the results on Type 1 of VRPSTW}

Taillard et al (1997) designed a TS heuristic for Type 1 of VRPSTW and tested it on the 56 benchmark problems. The results reported were feasible solutions to the VRPHTW in each case, that is, the percentage of non-violated time windows was $100 \%$, and the number of routes was set to the best solution reported in the literature for each problem, not minimized by the algorithm.

As in Taillard et al (1997), we suppose that $\alpha_{i}=0, \beta_{i}=100$ for all $i$. The real Euclidean distances between customers are used during the computations, whereas the final results are rounded to the second decimal. The best solutions produced by our unified TS algorithm during the course of multiple experiments are reported in Table 1 for all 56 test problems, using the format: number of routes/total travel distance, percentage of non-violated time windows. In the table, our best solutions are compared with those produced by Taillard et al (1997) for the VRPSTW and other algorithms reported in the literature for the VRPHTW, respectively, using the format: number of routes/total travel distance. In the table, a double asterisk ${ }^{* *}$ indicates that our algorithm has improved the best known solution (lower number of routes required or shorter total travel distance) and a single asterisk * means a tie with the best solution produced by Taillard et al (1997). When the percentage of non-violated time windows is $100 \%$, they are compared with those for the VRPHTW as well. An 'H' after ** indicates that our algorithm has improved the best published solution and after * means a tie with the best published solution for the VRPHTW. Overall, our algorithm has improved 25 solutions (12 cases with lower number of routes required, 13 cases with shorter total distance for the same number of routes required and non-violated time windows) and tied 16 solutions on the 56 test problems produced by Taillard et al (1997) for the VRPSTW, and improved three solutions and tied 16 best known solutions for the VRPHTW. Note that the method used by Taillard et al (1997) used the best known solution at that time for the number of vehicle routes and did not attempt to minimize the number of routes. The results from our algorithm also show that setting the time windows to be soft does obtain significant savings in the number of vehicles required and/or the total vehicle travelling distance for some routes.

The computation time in seconds for different sets of problems is shown in Table 2.

\section{Comparison of the results on Type 2 of VRPSTW}

Koskosidis et al (1992) developed an optimization-based heuristic for Type 2 of VRPSTW and tested it on the five sets of randomly generated problems and 21 instances of 56 Solomon's benchmark problems. The heuristic started with low penalty coefficients, which were gradually increased. In our algorithm, we suppose the penalty coefficients $\alpha_{i}=\beta_{i}=100$ for all $i$ and run it for the 21 instances listed. The comparison of the results is shown in Table 3, using the format: number of routes/total travel distance, percentage of non-violated time windows. The comparison of the CPU time in seconds is in Table 4.

Our algorithm has improved 12 and tied seven solutions on the 21 instances listed, indicated by ** and *, respectively. Among the 12 improved solutions, eight of them require a lower number of vehicle routes and four are of shorter total travel distance (for the same number of routes required and non-violated time windows). For the problem sets of C1, comparing with the heuristic of Koskosidis et al (1992) as well as our algorithm for Type 1 of VRPSTW, our algorithm for Type 2 of VRPSTW takes much more CPU time to find the same best known solutions, and cannot obtain the best known solutions in two instances.

\section{Comparison of the results on Type 3 of VRPSTW}

For Type 3 of VRPSTW, Balakrishnan (1993) described three simple heuristics, Chiang and Russell (2004) developed a TS solution method. The eight problems based on the R1 and $\mathrm{RC} 1$ sets of the Solomon benchmark problems were used to test the algorithms. The hard time windows in the original benchmark data were converted to soft time windows by allowing a certain percentage of time window violation, $E_{\max }=L_{\max }$, that is, $P_{\max }$, as in Balakrishnan (1993) and Chiang and Russell (2004). $P_{\max }\left(E_{\max }\right.$ and $\left.L_{\max }\right)$ and $W_{\max }$ were expressed as a percentage of the maximum allowable route time duration. $W_{\max }$ had the interesting effect of making some of the soft time window problems more difficult to solve than the original hard time window problems which specify no limit on wait time. To be consistent with Balakrishnan (1993) and Chiang and Russell (2004), the $P_{\max }\left(E_{\max }\right.$ and $\left.L_{\max }\right)$ and $W_{\max }$ values varied in combinations of 0,5 , and $10 \%$ of the maximum allowable route time duration, and the penalty coefficients $\alpha_{i}$ and $\beta_{i}$ were set equal to 1 . 
Table 1 Comparison of the results on Type 1 of VRPSTW

\begin{tabular}{|c|c|c|c|c|c|c|}
\hline \multirow[b]{2}{*}{ Problem } & \multicolumn{2}{|c|}{ VRPHTW } & \multicolumn{4}{|c|}{ VRPSTW } \\
\hline & Best published solution & Reference & Taillard et al & & et al & \\
\hline R101 & $19 / 1650.80$ & Rochat and Taillard (1995) & $19 / 1650.79$ & $14 / 1535.24$ & $75 \%$ & $* *$ \\
\hline $\mathrm{R} 102$ & $17 / 1486.12$ & Rochat and Taillard (1995) & $17 / 1487.60$ & $13 / 1416.84$ & $89 \%$ & $* *$ \\
\hline R103 & $13 / 1292.85$ & Homberger and Gehring (1999) & $13 / 1294.24$ & $11 / 1267.28$ & $96 \%$ & $* *$ \\
\hline R104 & $9 / 1013.32$ & Homberger and Gehring (1999) & $10 / 982.72$ & $9 / 983.50$ & $99 \%$ & $* *$ \\
\hline $\mathrm{R} 105$ & $14 / 1377.11$ & Rochat and Taillard (1995) & 14/1377.11 & $13 / 1441.24$ & $98 \%$ & $* *$ \\
\hline R106 & $12 / 1252.03$ & Rochat and Taillard (1995) & $12 / 1259.71$ & $11 / 1355.25$ & $97 \%$ & $* *$ \\
\hline R107 & $10 / 1112.16$ & Chiang and Russell (2004) & $10 / 1126.69$ & $10 / 1147.59$ & $100 \%$ & \\
\hline R108 & 9/964.38 & Cordeau et al (2001) & 9/968.59 & 9/978.69 & $100 \%$ & \\
\hline R109 & $11 / 1194.73$ & Homberger and Gehring (1999) & $11 / 1214.54$ & $11 / 1264.22$ & $100 \%$ & \\
\hline $\mathrm{R} 110$ & $10 / 1125.04$ & Cordeau et al (2001) & $11 / 1080.36$ & $11 / 1083.99$ & $100 \%$ & \\
\hline $\mathrm{R} 111$ & $10 / 1096.72$ & Rousseau et al (2002) & $10 / 1104.83$ & $10 / 1138.47$ & $100 \%$ & \\
\hline $\mathrm{R} 112$ & 9/982.14 & Gambardella et al (1999) & $10 / 964.01$ & $10 / 963.22$ & $100 \%$ & $* *$ \\
\hline $\mathrm{C} 101$ & $10 / 828.94$ & Rochat and Taillard (1995) & $10 / 828.94$ & $10 / 828.94$ & $100 \%$ & *H \\
\hline C102 & $10 / 828.94$ & Rochat and Taillard (1995) & $10 / 828.94$ & $10 / 828.94$ & $100 \%$ & ${ }^{*} \mathrm{H}$ \\
\hline C103 & $10 / 828.06$ & Rochat and Taillard (1995) & $10 / 828.06$ & $10 / 831.03$ & $100 \%$ & \\
\hline C104 & $10 / 824.78$ & Rochat and Taillard (1995) & $10 / 824.78$ & $10 / 824.78$ & $100 \%$ & ${ }^{*} \mathrm{H}$ \\
\hline $\mathrm{C} 105$ & $10 / 828.94$ & Rochat and Taillard (1995) & $10 / 828.94$ & $10 / 828.94$ & $100 \%$ & ${ }^{*} \mathrm{H}$ \\
\hline $\mathrm{C} 106$ & $10 / 828.94$ & Rochat and Taillard (1995) & $10 / 828.94$ & $10 / 828.94$ & $100 \%$ & ${ }^{*} \mathrm{H}$ \\
\hline C107 & $10 / 828.94$ & Rochat and Taillard (1995) & $10 / 828.94$ & $10 / 828.94$ & $100 \%$ & ${ }^{*} \mathrm{H}$ \\
\hline C108 & $10 / 828.94$ & Rochat and Taillard (1995) & $10 / 828.94$ & $10 / 828.94$ & $100 \%$ & ${ }^{*} \mathrm{H}$ \\
\hline C109 & $10 / 828.94$ & Rochat and Taillard (1995) & $10 / 828.94$ & $10 / 828.94$ & $100 \%$ & ${ }^{*} \mathrm{H}$ \\
\hline RC101 & $14 / 1697.43$ & Homberger and Gehring (1999) & $14 / 1696.94$ & $13 / 1654.30$ & $92 \%$ & $* *$ \\
\hline $\mathrm{RC} 102$ & $12 / 1558.07$ & Homberger and Gehring (1999) & $12 / 1554.75$ & $12 / 1593.71$ & $100 \%$ & \\
\hline $\mathrm{RC} 103$ & $11 / 1261.67$ & Shaw (1998) & $11 / 1264.27$ & $11 / 1321.71$ & $100 \%$ & \\
\hline RC104 & $10 / 1135.48$ & Cordeau et al (2001) & $10 / 1135.83$ & $10 / 1175.23$ & $100 \%$ & \\
\hline RC105 & $13 / 1637.15$ & Homberger and Gehring (1999) & $13 / 1643.38$ & $12 / 1654.07$ & $92 \%$ & $* *$ \\
\hline RC106 & $11 / 1427.13$ & Cordeau et al (2001) & $11 / 1448.26$ & $11 / 1422.72$ & $99 \%$ & \\
\hline RC107 & $11 / 1230.95$ & Rochat and Taillard (1995) & $11 / 1230.54$ & $11 / 1237.64$ & $100 \%$ & \\
\hline RC108 & $10 / 1147.26$ & Homberger and Gehring (1999) & $10 / 1139.82$ & $10 / 1184.55$ & $100 \%$ & \\
\hline R201 & $4 / 1252.37$ & Homberger and Gehring (1999) & $4 / 1254.80$ & $3 / 1500.36$ & $89 \%$ & $* *$ \\
\hline R202 & 3/1191.70 & Rousseau et al (2002) & $3 / 1214.28$ & $3 / 1205.79$ & $100 \%$ & $* *$ \\
\hline R203 & $3 / 942.64$ & Homberger and Gehring (1999) & $3 / 951.59$ & $3 / 950.36$ & $100 \%$ & $* *$ \\
\hline R204 & $2 / 838.36$ & Chiang and Russell (2004) & $2 / 941.76$ & $2 / 854.30$ & $100 \%$ & $* *$ \\
\hline R205 & 3/994.42 & Rousseau et al (2002) & $3 / 1038.72$ & $3 / 1001.75$ & $100 \%$ & $* *$ \\
\hline R206 & 3/906.14 & Schrimpf et al (2000) & $3 / 932.47$ & 3/917.94 & $100 \%$ & $* *$ \\
\hline $\mathrm{R} 207$ & $2 / 906.37$ & Chiang and Russell (2004) & $3 / 837.20$ & $2 / 903.01$ & $100 \%$ & ${ }^{* *} \mathrm{H}$ \\
\hline R208 & $2 / 731.23$ & Homberger and Gehring (1999) & 2/748.01 & $2 / 738.27$ & $100 \%$ & $* *$ \\
\hline R209 & $3 / 910.55$ & Homberger and Gehring (1999) & $3 / 959.47$ & 3/909.89 & $100 \%$ & ${ }^{* *} \mathrm{H}$ \\
\hline $\mathrm{R} 210$ & $3 / 955.39$ & Homberger and Gehring (1999) & $3 / 980.90$ & $3 / 948.20$ & $100 \%$ & ${ }^{* *} \mathrm{H}$ \\
\hline R211 & $2 / 909.29$ & Chiang and Russell (2004) & $2 / 923.80$ & $2 / 953.18$ & $100 \%$ & \\
\hline $\mathrm{C} 201$ & $3 / 591.56$ & Rochat and Taillard (1995) & $3 / 591.56$ & $3 / 591.56$ & $100 \%$ & $* \mathrm{H}$ \\
\hline C202 & $3 / 591.56$ & Rochat and Taillard (1995) & $3 / 591.56$ & $3 / 591.56$ & $100 \%$ & ${ }^{*} \mathrm{H}$ \\
\hline C203 & 3/591.17 & Rochat and Taillard (1995) & 3/591.17 & 3/591.17 & $100 \%$ & ${ }^{*} \mathrm{H}$ \\
\hline C204 & $3 / 590.60$ & Rochat and Taillard (1995) & $3 / 590.60$ & $3 / 590.60$ & $100 \%$ & ${ }^{*} \mathrm{H}$ \\
\hline C205 & $3 / 588.88$ & Rochat and Taillard (1995) & $3 / 588.88$ & $3 / 588.88$ & $100 \%$ & ${ }^{*} \mathrm{H}$ \\
\hline $\mathrm{C} 206$ & $3 / 588.49$ & Rochat and Taillard (1995) & $3 / 588.49$ & $3 / 588.49$ & $100 \%$ & ${ }^{*} \mathrm{H}$ \\
\hline C207 & $3 / 588.29$ & Rochat and Taillard (1995) & $3 / 588.29$ & $3 / 588.29$ & $100 \%$ & ${ }^{*} \mathrm{H}$ \\
\hline C208 & $3 / 588.32$ & Rochat and Taillard (1995) & $3 / 588.32$ & $3 / 588.32$ & $100 \%$ & ${ }^{*} \mathrm{H}$ \\
\hline RC201 & $4 / 1406.94$ & Cordeau et al (2001) & $4 / 1413.79$ & $4 / 1409.88$ & $100 \%$ & $* *$ \\
\hline RC202 & $3 / 1389.57$ & Homberger and Gehring (1999) & $4 / 1164.25$ & $3 / 1435.56$ & $100 \%$ & $* *$ \\
\hline RC203 & $3 / 1060.45$ & Homberger and Gehring (1999) & $3 / 1112.55$ & $3 / 1062.38$ & $100 \%$ & $* *$ \\
\hline RC204 & $3 / 799.32$ & Homberger and Gehring (1999) & $3 / 831.69$ & $3 / 799.98$ & $100 \%$ & $* *$ \\
\hline RC205 & $4 / 1302.42$ & Homberger and Gehring (1999) & $4 / 1328.21$ & $3 / 1656.80$ & $93 \%$ & $* *$ \\
\hline RC206 & $3 / 1160.91$ & Cordeau et al (2001) & $3 / 1158.81$ & $3 / 1186.80$ & $100 \%$ & \\
\hline RC207 & $3 / 1062.05$ & Cordeau et al (2001) & $3 / 1082.32$ & $3 / 1127.83$ & $100 \%$ & \\
\hline RC208 & $3 / 832.36$ & Cordeau et al (2001) & $3 / 847.90$ & $3 / 846.10$ & $100 \%$ & $* *$ \\
\hline
\end{tabular}


The computational results of our unified TS algorithm (UTS) and the comparison with the best solution found by Balakrishnan's simple heuristics (SIM) and Chiang and Russell's TS with advanced recovery (AR) are presented in Tables 5 and 6. Our algorithm solved all problem instances

Table 2 CPU time on Type 1 of VRPSTW

\begin{tabular}{lcc}
\hline Problem sets & CPU time in second & Average \\
\hline R1 & $313.08-1203.26$ & 786.34 \\
C1 & $18.68-238.92$ & 68.41 \\
RC1 & $190.65-1130.75$ & 697.25 \\
R2 & $140.77-897.37$ & 528.3 \\
C2 & $25.37-133.47$ & 56.97 \\
RC2 & $295.66-882.21$ & 548.73 \\
\hline
\end{tabular}

listed but one, while the simple heuristics left several problem instances unsolved. From the information given in the tables, our unified TS algorithm generally outperforms Balakrishnan's simple heuristics. Comparing the final solutions found by our TS algorithm with those by Chiang and Russell's TS, there are seven cases where our unified TS algorithm clearly gives a better solution with less total route distance travelled for the same number of vehicles used and $100 \%$ non-violated windows (denoted by ${ }^{* *}$ in Table 5); also there are 30 cases where our unified TS algorithm produces more non-violated windows for the same number of vehicles used (denoted by ${ }^{*}$ in Tables 5 and 6 ). In these 30 cases denoted by ${ }^{*}$, the improvement in the percentage of non-violated windows is generally at the expense of greater distance travelled; the best solution depends on the weightings used for these elements of the objective function.

Table 3 Comparison of the results on Type 2 of VRPSTW

\begin{tabular}{|c|c|c|c|c|c|}
\hline \multirow[t]{2}{*}{ Problem } & \multicolumn{2}{|c|}{ Koskosidis et al } & \multicolumn{3}{|c|}{ Fu et al } \\
\hline & K/distance & $\begin{array}{l}\text { Non-violated } \\
\text { windows }(\%)\end{array}$ & K/distance & $\begin{array}{l}\text { Non-violated } \\
\text { windows }(\%)\end{array}$ & \\
\hline R101 & $21 / 1856$ & 100 & $14 / 1872.94$ & 56 & $* *$ \\
\hline $\mathrm{R} 102$ & $19 / 1628$ & 100 & $13 / 1732.54$ & 71 & $* *$ \\
\hline R103 & $14 / 1428$ & 100 & $12 / 1542.79$ & 91 & $* *$ \\
\hline R104 & $10 / 1114$ & 100 & 10/1107.18 & 100 & $* *$ \\
\hline R108 & $10 / 975$ & 100 & $10 / 968.34$ & 100 & $* *$ \\
\hline R109 & $13 / 1244$ & 98 & $11 / 1379.87$ & 96 & $* *$ \\
\hline C101 & $10 / 829$ & 100 & $10 / 828.94$ & 100 & * \\
\hline C102 & $10 / 829$ & 100 & $10 / 828.94$ & 100 & $*$ \\
\hline C103 & $10 / 829$ & 100 & $10 / 918.08$ & 100 & \\
\hline C104 & $10 / 829$ & 100 & $10 / 899.00$ & 100 & \\
\hline C105 & $10 / 829$ & 100 & $10 / 828.94$ & 100 & * \\
\hline C106 & $10 / 829$ & 100 & $10 / 828.94$ & 100 & $*$ \\
\hline C107 & $10 / 829$ & 100 & $10 / 828.94$ & 100 & * \\
\hline C108 & $10 / 829$ & 100 & $10 / 828.94$ & 100 & $*$ \\
\hline C109 & $10 / 829$ & 100 & $10 / 828.94$ & 100 & $*$ \\
\hline RC101 & $16 / 1815$ & 95 & $13 / 1851.22$ & 74 & $* *$ \\
\hline RC102 & $14 / 1605$ & 94 & $13 / 1772.42$ & 99 & $* *$ \\
\hline RC103 & $13 / 1390$ & 100 & $11 / 1416.81$ & 100 & $* *$ \\
\hline RC104 & $10 / 1353$ & 100 & $10 / 1262.55$ & 100 & $* *$ \\
\hline RC106 & $13 / 1541$ & 92 & $12 / 1531.57$ & 99 & $* *$ \\
\hline RC108 & $11 / 1315$ & 99 & $11 / 1224.72$ & 100 & $* *$ \\
\hline
\end{tabular}

Table 4 Comparison of CPU time on Type 2 of VRPSTW

\begin{tabular}{lcccc}
\hline Algorithm & Problem sets & CPU time in seconds & Average & Computer \\
\hline Koskosidis et al & $\mathrm{R} 1$ & $209.1-903.3$ & 560.9 & 3.0 \\
& $\mathrm{C} 1$ & $2.9-3.3$ & 689.4 & IBM3081/VM370 \\
& $\mathrm{RC} 1$ & $570.0-834.7$ & 993.1 & $600 \mathrm{MHz}$ Pentium-II \\
Fu et al & $\mathrm{R} 1$ & $634.8-1357.3$ & 315.1 & PC with $184 \mathrm{MB}$ RAM \\
& $\mathrm{C} 1$ & $188.5-472.1$ & 810.5 & \\
\hline
\end{tabular}


Table 5 Comparison of the results on Type 3 of VRPSTW

\begin{tabular}{|c|c|c|c|c|c|c|c|c|c|c|c|c|c|c|c|c|}
\hline \multirow[t]{2}{*}{ Problem } & \multirow[t]{2}{*}{$\begin{array}{l}W_{\max }: \\
P_{\max }\left(E_{\max }, L_{\max }\right):\end{array}$} & \multicolumn{3}{|c|}{$\begin{array}{l}0 \\
0\end{array}$} & \multicolumn{3}{|c|}{$\begin{array}{l}5 \\
0\end{array}$} & \multicolumn{3}{|c|}{$\begin{array}{c}10 \\
0\end{array}$} & \multicolumn{3}{|c|}{$\begin{array}{l}0 \\
5\end{array}$} & \multicolumn{3}{|c|}{$\begin{array}{l}5 \\
5\end{array}$} \\
\hline & & $S I M$ & $A R$ & UTS & SIM & $A R$ & UTS & SIM & $A R$ & UTS & $S I M$ & $A R$ & UTS & $S I M$ & $A R$ & $U T S$ \\
\hline \multirow[t]{3}{*}{ R101 } & Number of vehicles used & & 22 & & 19 & 19 & 20 & 19 & 19 & 19 & 16 & 14 & 15 & 17 & 14 & 14 \\
\hline & Total route distance & & 2439 & & 2043 & 1808 & 1757 & 1915 & 1692 & 1695 & 1917 & 1483 & 1628 & 1903 & 1392 & 1456 \\
\hline & $\%$ Non-violated windows & & 100 & & 100 & 100 & 100 & 100 & 100 & 100 & 55 & 25 & 42 & 72 & 29 & $37^{*}$ \\
\hline \multirow[t]{3}{*}{ R102 } & Number of vehicles used & & 19 & 19 & 19 & 17 & 17 & 19 & 17 & 17 & 14 & 12 & 12 & 15 & 12 & 12 \\
\hline & Total route distance & & 1958 & $1685^{* *}$ & 1877 & 1600 & $1470^{* *}$ & 1890 & 1511 & $1490^{* *}$ & 1754 & 1364 & 1389 & 1693 & 1259 & 1348 \\
\hline & $\%$ Non-violated windows & & 100 & 100 & 100 & 100 & 100 & 100 & 100 & 100 & 69 & 39 & $61^{*}$ & 80 & 44 & $60^{*}$ \\
\hline \multirow[t]{3}{*}{ R103 } & Number of vehicles used & & 14 & 14 & & 13 & 14 & & 13 & 14 & 13 & 11 & 11 & 13 & 10 & 11 \\
\hline & Total route distance & & 1475 & $1381^{* *}$ & & 1370 & 1234 & & 1304 & 1109 & 1436 & 1126 & 1189 & 1530 & 1134 & 1232 \\
\hline & $\%$ Non-violated windows & & 100 & 100 & & 100 & 100 & & 100 & 100 & 77 & 60 & $78^{*}$ & 84 & 60 & 81 \\
\hline \multirow[t]{3}{*}{ R109 } & Number of vehicles used & 13 & 12 & 12 & 13 & 12 & 12 & 13 & 12 & 12 & 12 & 11 & 11 & 12 & 11 & 11 \\
\hline & Total route distance & 1567 & 1219 & $1206^{* *}$ & 1482 & 1172 & $1159^{* *}$ & 1492 & 1165 & $1158^{* *}$ & 1383 & 1123 & 1158 & 1363 & 1093 & 1140 \\
\hline & $\%$ Non-violated windows & 100 & 100 & 100 & 100 & 100 & 100 & 100 & 100 & 100 & 73 & 62 & $82 *$ & 80 & 58 & $82^{*}$ \\
\hline \multirow[t]{3}{*}{ Problem } & $W_{\max }:$ & & 10 & & & 0 & & & 5 & & & 10 & & & & \\
\hline & $P_{\max }\left(E_{\max }, L_{\max }\right):$ & & 5 & & & 10 & & & 10 & & & 10 & & & & \\
\hline & & SIM & $A R$ & UTS & SIM & $A R$ & UTS & SIM & $A R$ & UTS & SIM & $A R$ & UTS & & & \\
\hline \multirow[t]{3}{*}{ R101 } & Number of vehicles used & 17 & 14 & 14 & 14 & 12 & 12 & 15 & 12 & 12 & 15 & 12 & 12 & & & \\
\hline & Total route distance & 1885 & 1370 & 1438 & 1737 & 1266 & 1399 & 1832 & 1216 & 1364 & 1832 & 1212 & 1376 & & & \\
\hline & $\%$ Non-violated windows & 72 & 24 & $45^{*}$ & 44 & 14 & $33^{*}$ & 62 & 11 & $37^{*}$ & 62 & 8 & $31^{*}$ & & & \\
\hline \multirow[t]{3}{*}{ R102 } & Number of vehicles used & 15 & 12 & 12 & 13 & 11 & 11 & 14 & 11 & 11 & 14 & 10 & 11 & & & \\
\hline & Total route distance & 1636 & 1265 & 1339 & 1507 & 1167 & 1324 & 1790 & 1147 & 1272 & 1569 & 1173 & 1287 & & & \\
\hline & $\%$ Non-violated windows & 83 & 47 & $61^{*}$ & 63 & 35 & $55^{*}$ & 78 & 39 & $56^{*}$ & 81 & 33 & 51 & & & \\
\hline \multirow[t]{3}{*}{ R103 } & Number of vehicles used & 13 & 11 & 11 & 12 & 10 & 10 & 13 & 10 & 10 & 13 & 10 & 10 & & & \\
\hline & Total route distance & 1452 & 1066 & 1168 & 1363 & 1028 & 1209 & 1575 & 1008 & 1197 & 1657 & 1013 & 1185 & & & \\
\hline & $\%$ Non-violated windows & 86 & 59 & $73^{*}$ & 68 & 57 & $76^{*}$ & 82 & 56 & $75^{*}$ & 83 & 58 & $76^{*}$ & & & \\
\hline \multirow[t]{3}{*}{ R109 } & Number of vehicles used & 13 & 11 & 11 & 11 & 10 & 11 & 12 & 10 & 11 & 12 & 10 & 11 & & & \\
\hline & Total route distance & 1445 & 1084 & 1168 & 1311 & 1017 & 1161 & 1431 & 1019 & 1176 & 1431 & 1005 & 1183 & & & \\
\hline & $\%$ Non-violated windows & 95 & 60 & $75^{*}$ & 67 & 54 & $85^{*}$ & 90 & 47 & 82 & 90 & 47 & 82 & & & \\
\hline
\end{tabular}


Table 6 Comparison of the results on Type 3 of VRPSTW

\begin{tabular}{|c|c|c|c|c|c|c|c|c|c|c|c|c|c|c|c|c|}
\hline \multirow[t]{2}{*}{ Problem } & \multirow[t]{2}{*}{$\begin{array}{l}W_{\max }: \\
P_{\max }\left(E_{\max }, L_{\max }\right):\end{array}$} & \multicolumn{3}{|c|}{$\begin{array}{l}0 \\
0\end{array}$} & \multicolumn{3}{|c|}{$\begin{array}{l}5 \\
0\end{array}$} & \multicolumn{3}{|c|}{$\begin{array}{c}10 \\
0\end{array}$} & \multicolumn{3}{|c|}{$\begin{array}{l}0 \\
5\end{array}$} & \multicolumn{3}{|c|}{$\begin{array}{l}5 \\
5\end{array}$} \\
\hline & & $S I M$ & $A R$ & UTS & $S I M$ & $A R$ & $U T S$ & $S I M$ & $A R$ & $U T S$ & $S I M$ & $A R$ & UTS & $S I M$ & $A R$ & UTS \\
\hline \multirow[t]{3}{*}{ RC101 } & Number of vehicles used & 16 & 5 & 16 & 16 & 15 & 15 & 16 & 15 & 15 & 14 & 12 & 13 & 15 & 13 & 13 \\
\hline & Total route distance & 2200 & 1764 & 1913 & 2012 & 1643 & 1704 & 2012 & 1651 & 1685 & 1835 & 1522 & 1594 & 1972 & 1425 & 1554 \\
\hline & $\%$ Non-violated windows & 100 & 100 & 100 & 100 & 100 & 100 & 100 & 100 & 100 & 68 & 47 & 72 & 94 & 44 & $72^{*}$ \\
\hline \multirow{3}{*}{ RC102 } & Number of vehicles used & & 14 & 14 & 14 & 13 & 14 & 14 & 13 & 14 & 13 & 12 & 12 & 14 & 11 & 11 \\
\hline & Total route distance & & 1627 & 1675 & 1807 & 1560 & 1617 & 1808 & 1530 & 1502 & 1679 & 1368 & 1523 & 1776 & 1357 & 1475 \\
\hline & $\%$ Non-violated windows & & 100 & 100 & 100 & 100 & 100 & 100 & 100 & 100 & 84 & 60 & $79^{*}$ & 93 & 61 & $74^{*}$ \\
\hline \multirow[t]{3}{*}{ RC103 } & Number of vehicles used & 13 & 11 & 12 & 12 & 11 & 12 & 12 & 11 & 12 & 12 & 10 & 11 & 13 & 10 & 11 \\
\hline & Total route distance & 1885 & 1362 & 1428 & 1676 & 1296 & 1358 & 1679 & 1284 & 1331 & 1605 & 1229 & 1265 & 1680 & 1186 & 1251 \\
\hline & $\%$ Non-violated windows & 100 & 100 & 100 & 100 & 100 & 100 & 100 & 100 & 100 & 88 & 79 & 90 & 97 & 69 & 89 \\
\hline \multirow{3}{*}{ RC106 } & Number of vehicles used & 13 & 12 & 13 & & 12 & 12 & & 12 & 12 & 13 & 11 & 11 & 13 & 11 & 11 \\
\hline & Total route distance & 1664 & 1424 & 1492 & & 1409 & 1420 & & 1409 & 1414 & 1620 & 1269 & 1329 & 1699 & 1233 & 1325 \\
\hline & $\%$ Non-violated windows & 100 & 100 & 100 & & 100 & 100 & & 100 & 100 & 95 & 71 & $82^{*}$ & 98 & 58 & $77^{*}$ \\
\hline \multirow[t]{3}{*}{ Problem } & $W_{\max }:$ & & 10 & & & 0 & & & 5 & & & 10 & & & & \\
\hline & $P_{\max }\left(E_{\max }, L_{\max }\right):$ & & 5 & & & 10 & & & 10 & & & 10 & & & & \\
\hline & & $S I M$ & $A R$ & $U T S$ & SIM & $A R$ & $U T S$ & $S I M$ & $A R$ & UTS & $S I M$ & $A R$ & UTS & & & \\
\hline \multirow[t]{3}{*}{ RC101 } & Number of vehicles used & 14 & 13 & 13 & 14 & 11 & 12 & 14 & 11 & 12 & 15 & 11 & 12 & & & \\
\hline & Total route distance & 1839 & 1424 & 1529 & 1784 & 1305 & 1502 & 1795 & 1288 & 1474 & 1832 & 1275 & 1457 & & & \\
\hline & $\%$ Non-violated windows & 56 & 39 & $64^{*}$ & 60 & 25 & 59 & 61 & 36 & 59 & 62 & 27 & 54 & & & \\
\hline \multirow[t]{3}{*}{$\mathrm{RC} 102$} & Number of vehicles used & 13 & 11 & 12 & 14 & 11 & 11 & 13 & 11 & 11 & 14 & 11 & 11 & & & \\
\hline & Total route distance & 1850 & 1375 & 1413 & 2060 & 1249 & 1503 & 1719 & 1218 & 1458 & 1569 & 1222 & 1367 & & & \\
\hline & $\%$ Non-violated windows & 88 & 58 & 81 & 97 & 55 & $69^{*}$ & 83 & 56 & $78^{*}$ & 81 & 56 & $74^{*}$ & & & \\
\hline \multirow[t]{3}{*}{$\mathrm{RC} 103$} & Number of vehicles used & 12 & 10 & 11 & 12 & 10 & 10 & 12 & 10 & 11 & 13 & 10 & 11 & & & \\
\hline & Total route distance & 1469 & 1183 & 1254 & 1571 & 1137 & 1258 & 1530 & 1123 & 1266 & 1657 & 1119 & 1275 & & & \\
\hline & $\%$ Non-violated windows & 82 & 69 & 86 & 92 & 65 & $86^{*}$ & 92 & 63 & 87 & 83 & 65 & 90 & & & \\
\hline \multirow[t]{3}{*}{$\mathrm{RC} 106$} & Number of vehicles used & 12 & 11 & 11 & 13 & 10 & 11 & 13 & 10 & 11 & 12 & 10 & 11 & & & \\
\hline & Total route distance & 1496 & 1223 & 1336 & 1620 & 1191 & 1301 & 1620 & 1158 & 1303 & 1431 & 1160 & 1337 & & & \\
\hline & $\%$ Non-violated windows & 71 & 61 & $81^{*}$ & 97 & 47 & 77 & 97 & 50 & 73 & 90 & 49 & 81 & & & \\
\hline
\end{tabular}


Table 7 Comparison of CPU time on Type 3 of VRPSTW

\begin{tabular}{lccc}
\hline Algorithm & $\begin{array}{c}\text { Problem } \\
\text { sets }\end{array}$ & $\begin{array}{c}\text { CPU time in } \\
\text { seconds }\end{array}$ & Computer \\
\hline SIM & R1 & $18.9-79.1$ & \\
& RC1 & $17.6-55.1$ & $25 \mathrm{MHz} 80386 / 387$ \\
AR & R1 & $448.2-692.4$ & \\
& RC1 & $595.8-844.8$ & 2.25 to GHz Athlon \\
UTS & RI & $170.0-1586.3$ & 600 MHz Pentium-II PC \\
& RCI & $193.1-1899.7$ & with 184 MB RAM \\
\hline
\end{tabular}

The computational requirements for TS are greater than the simple heuristics, as shown in Table 7.

\section{Conclusions}

The VRPSTW is an extension of the basic VRP and may arise in a variety of applications. The different forms of time window violation allowed lead to different types of VRPSTW. The existing approaches in the literature are usually designed for a special type of VRPSTW. In this paper, the differences and relationships between six main types of VRPSTW are discussed. Then a unified penalty function and a unified TS algorithm for these main types of VRPSTW is proposed, with which a given type of VRPSTW can be solved by simply setting appropriate values for the corresponding parameters in the penalty function. The distinctive features of this TS algorithm are the use of a mixed neighbourhood structure based on the 2-interchange generation mechanism, the allowance of the search process to examine solutions that may be infeasible with respect to the capacity and duration constraints, and the use of stochastic diversification in the selection of the neighbourhood moves and the tabu length. Finally, to test the computational performance of the algorithm, we ran it on the benchmark problems and compared the results with other methods for three types of VRPSTW in the literature. It showed that our algorithm has improved many best known solutions for the benchmark problems.

Acknowledgements - We are grateful to the anonymous referees for their useful comments and suggestions that helped us to improve the presentation of this paper. This research was supported by the National Natural Science Foundation of China (NSFC, 70071003, 70671108).

\section{References}

Balakrishnan N (1993). Simple heuristic for the vehicle routing problem with soft time windows. J Opl Res Soc 44: 279-287.

Brandão J (2004). A tabu search algorithm for the open vehicle routing problem. Eur J Opl Res 157: 552-564.

Chiang W-C and Russell RA (2004). A metaheuristic for the vehiclerouting problem with soft time windows. J Opl Res Soc 55: 1298-1310.
Cordeau J-F, Laporte G and Mercier A (2001). A unified tabu search heuristic for vehicle routing problems with time windows. $J \mathrm{Opl}$ Res Soc 52: 928-936.

Cordeau J-F, Desaulniers G, Desrosiers J, Solomon MM and Soumis F (2002). VRP with time windows. In: Toth $P$ and Vigo D (eds). The Vehicle Routing Problem. SIAM Monographs on Discrete Mathematics and Applications. SIAM: Philadelphia PA, pp 157-194.

Duhamel C, Potvin JY and Rousseau JM (1997). A tabu search heuristic for the vehicle routing problem with backhauls and time windows. Transport Sci 31: 49-59.

Fagerholt K (2001). Ship scheduling with soft time windows: An optimisation based approach. Eur J Opl Res 131: 559-571.

Fu Z, Eglese R and Li LYO (2005). A new tabu search heuristic for the open vehicle routing problem. J Opl Res Soc 56: 267-274.

Fu Z, Eglese R and Li LYO (2006). Corrigendum: A new tabu search heuristic for the open vehicle routing problem. J Opl Res Soc 57: $1018-1018$.

Gambardella LM, Taillard E and Agazzi G (1999). MACS-VRPTW: A multiple ant colony system for vehicle routing problems with time windows. In: Corne D, Dorigo M and Glover F (eds). New Ideas in Optimization. McGraw-Hill: London, pp 63-76.

Gendreau M, Hertz A and Laporte G (1994). A tabu search heuristic for the vehicle routing problem. Mngt Sci 40: 1276-1290.

Glover F and Laguna M (1997). Tabu Search. Kluwer Academic Publishers: Norwell, MA.

Homberger J and Gehring H (1999). Two evolutionary metaheuristics for the vehicle routing problem with time windows. INFOR 37: 297-318.

Koskosidis YA, Powell WB and Solomon MM (1992). An optimization-based heuristic for vehicle routing and scheduling with soft time windows constraints. Transport Sci 26: 69-85.

Osman IH (1993). Metastrategy simulated annealing and tabu search algorithms for the vehicle routing problem. Ann Opns Res 41: 421-451.

Pureza VM and França PM (1991). Vehicle routing problems via tabu search metaheuristic. Technical Report CRT-347, Centre for Research on Transportation, Montreal, Canada.

Rochat Y and Taillard ED (1995). Probabilistic diversification and intensification in local search for vehicle routing. J Heuristics 1: $147-167$.

Rousseau LM, Gendreau M and Pesant G (2002). Using constraintbased operators to solve the vehicle routing problem with time windows. J Heuristics 1: 43-58.

Schrimpf G, Schneider J, Stamm-Wilbrandt H and Dueck G (2000). Record breaking optimization results using the ruin and recreate principle. J Comput Phys 159: 139-171.

Shaw P (1998). Using constraint programming and local search methods to solve vehicle routing problems. In: Maher M and Puget J-F (eds). Principles and Practice of Constraint ProgrammingCP98, Lecture Notes in Computer Science. Springer-Verlag: New York, pp 417-431.

Solomon MM (1987). Algorithms for the vehicle routing and scheduling problems with time window constraints. Opns Res 35: 254-265.

Taillard E, Badeau P, Gendreau M, Guertin F and Potvin JY (1997). A tabu search heuristic for the vehicle routing problem with soft time windows. Transport Sci 31: 170-186.

Van Breedam A (2001). Comparing descent heuristics and metaheuristics for the vehicle routing problem. Comput Opns Res 28: $289-315$.

Received June 2005; accepted October 2006 after two revisions 